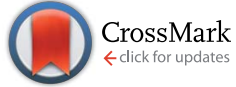

Cite this: J. Mater. Chem. A, 2016, 4, 15647

Received 29th June 2016

Accepted 14th September 2016

DOI: $10.1039 / c 6 t a 05455 b$

www.rsc.org/MaterialsA

\section{Designing intrinsically photostable low band gap polymers: a smart tool combining EPR spectroscopy and DFT calculations $\uparrow$}

\author{
Hugo Santos Silva, ${ }^{a}$ Isabel Fraga Domínguez, ${ }^{\text {bde }}$ Anthony Perthué, ${ }^{\text {bd }}$ Paul D. Topham, ${ }^{f}$ \\ Pierre-Olivier Bussière, ${ }^{\text {cd }}$ Roger C. Hiorns, ${ }^{\text {ag }}$ Christian Lombard, ${ }^{\text {hij }}$ Agnès Rivaton, ${ }^{\text {bd }}$ \\ Didier Bégué ${ }^{\star a}$ and Brigitte Pépin-Donat ${ }^{\star h i j}$
}

\begin{abstract}
A rapid and efficient method to identify the weak points of the complex chemical structure of low band gap (LBG) polymers, designed for efficient solar cells, when submitted to light exposure is reported. This tool combines Electron Paramagnetic Resonance (EPR) using the 'spin trapping method' coupled with density functional theory modelling (DFT). First, the nature of the short life-time radicals formed during the early-stages of photo-degradation processes are determined by a spin-trapping technique. Two kinds of short life-time radical ( $\mathrm{R}^{*}$ and $\left.\mathrm{R}^{\prime} \mathrm{O}^{*}\right)$ are formed after 'short-duration' illumination in an inert atmosphere and in ambient air, respectively. Second, simulation allows the identification of the chemical structures of these radicals revealing the most probable photochemical process, namely homolytical scission between the $\mathrm{Si}$ atom of the conjugated skeleton and its pendent side-chains. Finally, DFT calculations confirm the homolytical cleavage observed by EPR, as well as the presence of a group that is highly susceptible to photooxidative attack. Therefore, the synergetic coupling of a spin trapping method with DFT calculations is shown to be a rapid and efficient method for providing unprecedented information on photochemical mechanisms. This approach will allow the design of LBG polymers without the need to trial the material within actual solar cell devices, an often long and costly screening procedure.
\end{abstract}

\section{Introduction}

Incorporating stable materials is a prerequisite in creating long-lasting, stable devices. This is particularly pertinent for materials used to make organic solar cells because polymers used in the active layer are generally unstable under light exposure. Only very few photo-induced defects formed within the polymer are required to drastically impair solar cell

${ }^{a}$ Université de Pau et des Pays de l'Adour, IPREM (CNRS-UMR 5254), 2 Avenue Président Angot, 64053 Pau, France. E-mail: didier.begue@univ-pau.fr

${ }^{b}$ Université Blaise Pascal, Institut de Chimie de Clermont-Ferrand, Équipe Photochimie, BP 10448, F-63000 Clermont-Ferrand, France

${ }^{c}$ Université Clermont Auvergne, SIGMA Clermont, Institut de Chimie de ClermontFerrand, BP 10448, F-63000 Clermont-Ferrand, France

${ }^{d}$ CNRS, UMR 6296, ICCF, Équipe Photochimie, BP 80026, F-63171 Aubière, France ${ }^{e}$ Chemical Engineering and Applied Chemistry, Aston University, Birmingham, B4 7ET, $U K$

${ }^{f}$ Aston Materials Centre, Aston University, Birmingham, B4 7ET, UK

${ }^{g}$ CNRS, IPREM (EPCP, CNRS-UMR 5254), 64053 Pau, France

${ }^{h}$ Université Grenoble Alpes, INAC-SPRAM, F-38000 Grenoble, France

${ }^{i} C N R S$, INAC-SPRAM, F-38000 Grenoble, France

${ }^{j} C E A$, INAC-SPRAM, F-38000 Grenoble, France. E-mail: brigitte.pepin-donat@cea.fr

$\dagger$ Electronic supplementary information (ESI) available. See DOI: 10.1039/c6ta05455b performance, since such defects deleteriously affect the charge generation process. ${ }^{1}$

Besides photovoltaic conversion, other photophysical or photochemical processes can occur in the excited state. Among these latter processes, rearrangement, chain scission, and crosslinking all result in modification of the chemical structure of the polymer (see Scheme S1 in ESI $\dagger$ ). ${ }^{2}$ Identifying polymers with simple structures and even more troublesome for low band gap (LBG) polymers, which are now extensively used in efficient organic solar cells. ${ }^{3,4}$ Nevertheless, it is essential to design improved, efficient and stable LBG polymer structures to enable the fabrication of stable organic solar cells. The conjugated skeleton of LBG polymers is based on the alternation of donor and acceptor units on which alkyl side chains can be attached, where the attachments themselves may contain different types of heteroatoms. The alkyl side chains can be linear or branched, and be attached to the donor and/or acceptor units of the polymer. Based on these multiple combinations, countless types of LBG polymers can be synthesized. ${ }^{5,6}$

One of the main problems in addressing photodegradation is that there are no sufficiently sensitive tools that are able to detect at low concentrations both small structural changes in the the photodegradation mechanism(s) occurring is not easy for 
polymer (at the molecular scale) and the primary species at the origin of the photochemical process. In fact, a dramatic decrease in organic solar cell efficiency is generally associated with only very slight changes in the 'usual' spectrometric parameters (Raman, UV-visible, XPS...) of the polymer. ${ }^{7}$ Furthermore, such spectroscopies cannot reveal the primary species at the origin of degradation. They only allow detection of the structural/chemical changes due to subsequent sequential steps of the degradation process. Thus, they do not give information on the mechanism behind such processes. ${ }^{8}$ It is therefore vital to develop a technique that is able to unambiguously detect, and identify, the primary species involved in photochemical processes. Electronic Paramagnetic Resonance (EPR) combines simple implementation of global tracing methods (a few milligrams of the macroscopic sample deposited on the same substrate, under the same conditions as those in real organic solar cells, are directly observed) with very high sensitivity. ${ }^{9}$ In addition, the more sophisticated 'EPR spin trapping method' allows the detection of primary unstable free radicals resulting from direct (intrinsic) photochemical processes. Density Functional Theory (DFT) calculations allow one to identify weak points in a chemical structure in terms of their photochemical reactivity (via the binding energies of different bonds). Herein, we exploit the remarkable synergy of EPR spectroscopy and DFT modelling to obtain information on photochemical mechanisms, ${ }^{\mathbf{1 0 , 1 1}}$ thus allowing the design of push-pull polymers adapted to long-life organic solar cells without, most importantly, the necessity of testing them in real devices.

In the work herein, the more sophisticated 'Spin Trap Method' has been used in place of 'basic' EPR. The 'Spin Trap Method' consists of detecting radicals of short life-time (in this case the radicals eventually formed during the polymer photodegradation process) by trapping them with a diamagnetic molecule to produce a stable paramagnetic adduct; it was developed in parallel in 1968 by Janzen ${ }^{\mathbf{1 2 , 1 3}}$ and Lagercrantz. ${ }^{\mathbf{1 4}}$ This method has been used recently to study the formation of the oxygen superoxide anion radical, which is trapped at the very beginning of illumination and assumed to be responsible for the degradation of poly(3-hexylthiophene) (P3HT) in chlorobenzene in the presence of oxygen. ${ }^{15}$ In this study, the authors did not report radical trapping arising from polymer degradation. On closer inspection of their data, and in agreement with the authors, only $\mathrm{OH}^{*}$ radicals were trapped at the beginning of the experiment. However, after a few minutes of irradiation, a dramatic change was observed in the EPR spectrum. We ascribe this to the trapping of other radicals, such as those arising from degradation of the polymer. Therefore, the present study focuses on what happens after 15 minutes of illumination by solar simulated light in order to detect eventual radicals arising from the intrinsic polymer photochemical processes with the aim of further probing polymer photo-degradation mechanisms.

Poly[4,4'-bis(2-ethylhexyl)dithieno[3,2-b:2', $\left.3^{\prime}-d\right]$ silole]-2,6-diylalt-[2,5-bis(3-tetradecylthiophen-2-yl)thiazole-[5,4- $d]$ thiazole-1,8diyl] (PDTSTzTz) has been chosen as model LBG polymer, as its chemical structure covers most scenarios of low band gap polymers, see Fig. 1. The skeleton of PDTSTzTz comprises

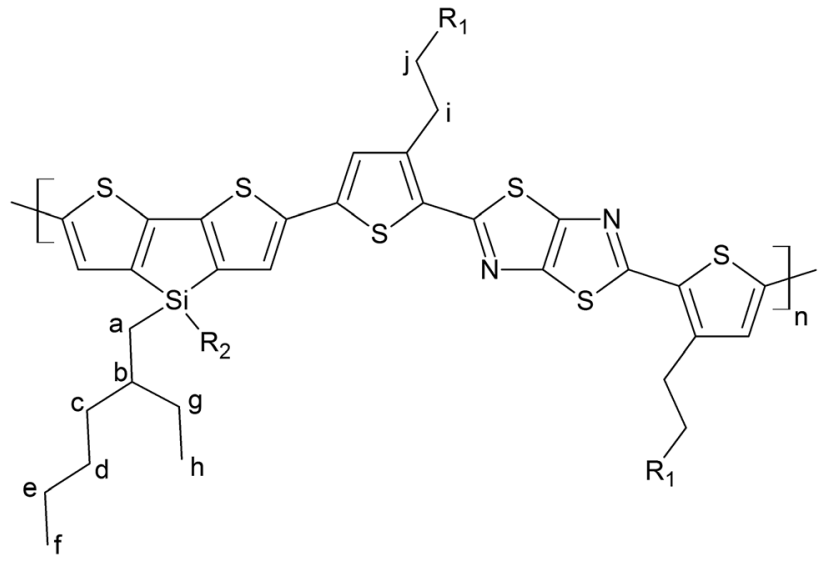

Fig. 1 Chemical structure of PDTSTzTz, where $R_{1}$ is a dodecyl linear chain and $R_{2}$ is a 2-ethylhexyl chain. The calculations were performed on a monomer of this structure for which $R_{1}$ is a methyl group. The hydrogen atoms used for the determination of the bond dissociation energies have been labelled $a-i$.

a 'push' dithenosilole (DTS) unit and a 'pull' thiazolothiazol (TzTz) unit; it has also two different side-chains, - one linear, one branched - with the branched side chains being attached to the conjugated backbone by an heteroatom.

\section{Materials and methods}

\subsection{Materials}

PDTSTzTz $\left(M_{\mathrm{n}}=45 \mathrm{~kg} \mathrm{~mol}^{-1}, D=3.5\right)$ was provided by Merck Chemicals and was used without further purification (see Fig. 1 for its chemical structure). Ortho-Dichlorobenzene and paraxylene were obtained from SIGMA-ALDRICH, HPLC grade (99\%). 5,5-Dimethyl-1-pyrroline $N$-oxide (DMPO) was obtained from SIGMA-Aldrich $(\geq 97 \%)$. Ethanol was obtained from SIGMA-Aldrich (99.9\%).

\subsection{Methods}

2.2.1 Sample preparation. Films on $\mathrm{KBr}$ substrates were prepared via doctor blading (Erichsen Coatmaster $809 \mathrm{MC}$ ) from a $0.9 \%(\mathrm{w} / \mathrm{w})$ polymer solution in para-xylene : orthodichlorobenzene (7:1). Films on PET/ITO substrates were dropcast from a $1 \%$ polymer solution $(\mathrm{w} / \mathrm{w})$ in ortho-dichlorobenzene. For the purpose of the EPR experiments, polymer films on PET/ITO were introduced in EPR Quartz tubes $(\varnothing=3 \mathrm{~mm})$ and wetted by a solution of DMPO in ethanol $\left(50 \mathrm{~g} \mathrm{~L}^{-1}\right)$.

2.2.2 UV-vis and IR monitoring before and after irradiation. UV-vis absorption spectra were obtained using a Shimadzu UV-2600 spectrophotometer with an integration sphere. Infrared transmission spectra were recorded by a Nicolet 760 Magna spectrophotometer purged with dry air (32 acquisitions with $4 \mathrm{~cm}^{-1}$ resolution). Irradiation of deposits on $\mathrm{KBr}$ substrates was performed in a Suntest CPS/XLS Atlas device, provided with a xenon lamp from ATLAS (NXE1700) configured at $750 \mathrm{~W} \mathrm{~m}^{-2}$ in the UV-visible domain. A filter cuts off IR irradiation and UV photons below $300 \mathrm{~nm}$. A cryostat maintained the Black Standard Temperature (BST) at $60{ }^{\circ} \mathrm{C}$, corresponding to a chamber temperature of $35{ }^{\circ} \mathrm{C}$. Samples irradiated in the absence of 


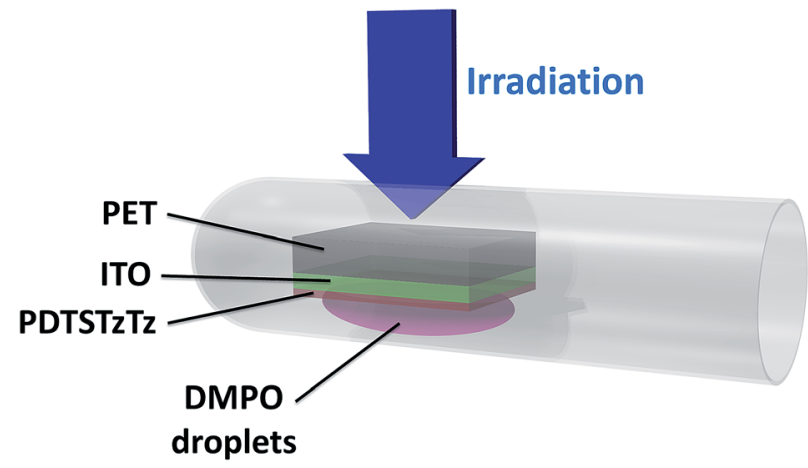

Fig. 2 Experimental set-up related to sample irradiation for EPR spintrapping analysis.

oxygen were encapsulated in borosilicate tubes under secondary vacuum $\left(10^{-6} \mathrm{~Pa}\right)$.

2.2.3 EPR analysis before and after illumination. EPR spectra were recorded on an EMX Brucker spectrometer operating at $9 \mathrm{GHz}$ at room temperature. They were simulated with free winsim 2002 software. ${ }^{16}$ EPR tubes containing polymer films deposited on PET/ITO were placed in a fixed position located $5 \mathrm{~cm}$ below the device producing the photons from the solar simulator (so that the center of the simulator corresponded to the center of the sample). The solar simulator was a New Port Oriel, Sol 3A class 3A solar simulator 94023A. Irradiation of the sample was made according to the set-up depicted in Fig. 2.

In the configuration shown in Fig. 2, the sample was exposed to 15 min AM 1.5 light soaking and the substrate was firstly irradiated before the deposited polymer was wetted by the DMPO solution in order to minimize photo-degradation of the DMPO. EPR spectra were recorded under Ar atmosphere after air exchange inside a glovebox in order to avoid broadening of the line widths due to the presence of oxygen.

2.2.4 Computational details. The photostability of the polymer was evaluated via calculation of the bond dissociation energies of selected bonds in the lateral chains of a DTSTzTz monomer, namely the $\mathrm{C}-\mathrm{H}$ bonds in both lateral chains (labels a-j in Fig. 1) and the Si-C bond or $\mathrm{C}-\mathrm{C}$ bonds involved in the attachment of the side chains to the polymer. This was calculated within B3LYP/6-31G** level of theory ${ }^{17-22}$ using UKS wavefunctions whenever needed. The general system studied is depicted in Fig. 1. The procedure used to evaluate the lability of these hydrogen atoms has been described elsewhere. ${ }^{23}$ The $g$-factors were calculated on the B3LYP/6-31G** optimized geometries but using the B3LYP/IGLO-III ${ }^{24}$ level of theory using an UKS wave-function. All calculations were performed on ORCA 3.0.3 software. ${ }^{25}$

\section{Results and discussion}

\subsection{Results}

3.1.1 UV-vis and IR spectroscopy. Significant absorbance decrease in the UV-vis spectra of PDTSTzTz could be detected when exposed to light (AM 1.5) under ambient air conditions even after very short irradiation times ( $\sim 2 \%$ decrease after $15 \mathrm{~min}$ exposure) (see Fig. 3). This indicates a photobleaching of the polymer resulting from the disruption of the $\pi$-conjugation of the backbone (e.g. via breakup or saturation of the backbone).

It is also clear that when irradiation is performed in the absence of oxygen, the effect of photodegradation on the UV-visible absorbance is significantly reduced compared to irradiation in ambient air, as previously evidenced for MDMO-PPV, P3HT and PCDTBT. ${ }^{26}$ In parallel, IR spectroscopy revealed a decrease of the polymer bands and the formation of oxidation products upon irradiation in the presence of air, whereas no new bands were observed when samples were irradiated in glass tubes sealed under vacuum, confirming that the tubes were fully degassed (see Fig. 3b).

It is noteworthy that photooxidative processes occurring with irradiation in ambient air, resulting in easily identifiable oxygen fixation on the chemical structure, does not exclude the involvement of pure photochemical processes that are

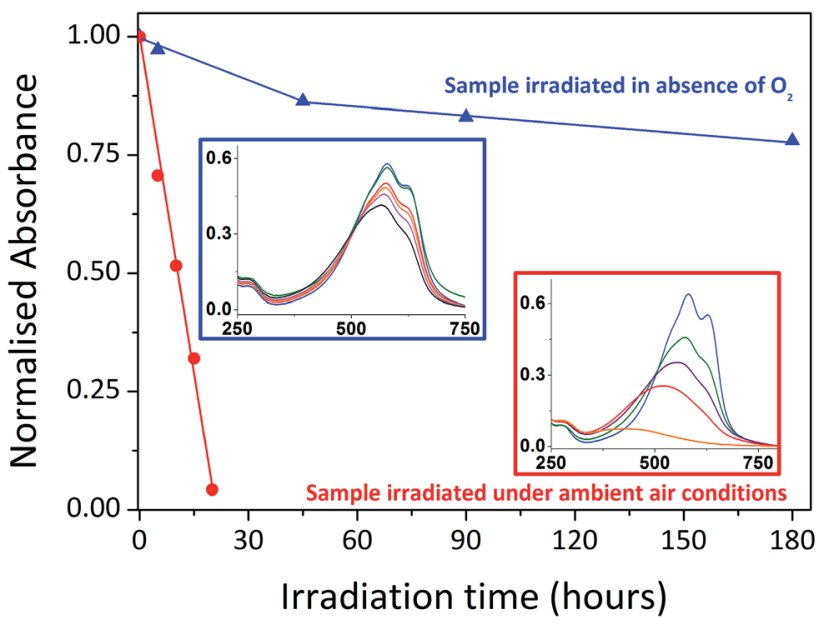

(a)

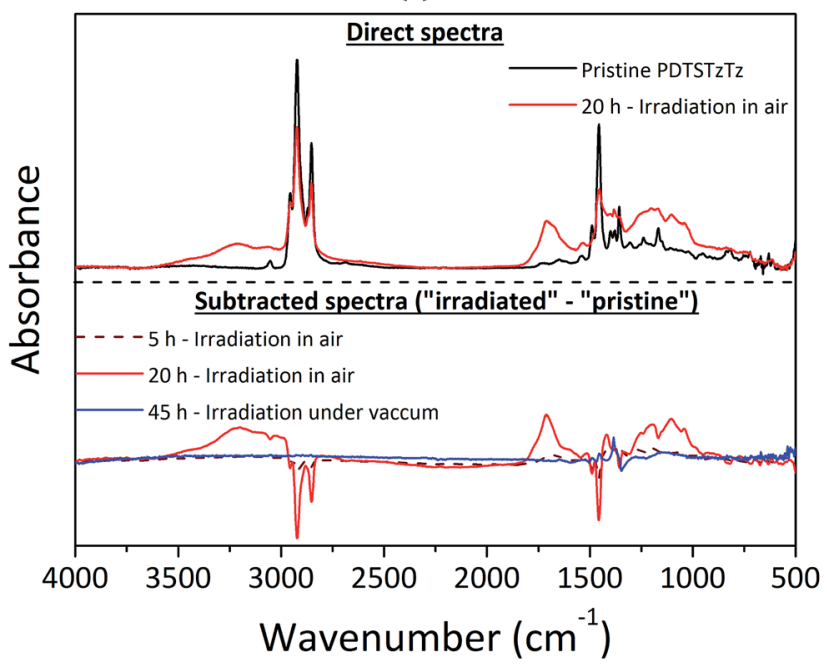

(b)

Fig. 3 Changes in (a) UV-visible and (b) IR spectra of PDTSTzTz thin films during irradiation in the SUNTEST device under ambient conditions (red) and in the absence of oxygen (blue). 
unaffected by atmospheric conditions. Furthermore, if an efficient encapsulation barrier may suppress, or at least drastically reduce, oxidation processes, pure photochemical processes have to be regarded as the intrinsic fragility of the polymer. Therefore, characterizing exclusively light-induced degradation processes is crucial if one wishes to develop intrinsically more stable materials, which will then allow significant improvements in device lifetime to be made. The spectroscopic analysis of PDTSTzTz deposits submitted to long duration irradiations in the total absence of oxygen reveals the presence of intrinsic photochemical processes that lead to some modification of the PDTSTzTz backbone structure. In this respect, previous work devoted to other polymers, such as P3HT and PCDTBT, suggested that irradiation in the absence of oxygen provoked homolytical scission between the side chain and conjugated backbone. Successive saturation of the P3HT conjugated skeleton by the alkyl fragments would induce a reduction of the conjugation length of the $\pi$-conjugated system, thus explaining the decrease in absorbance. ${ }^{27}$ Macroradical recombination was proposed in the case of PCDTBT, with parallel formation of low molecular weight alkyl products. ${ }^{9}$ Moreover, to our knowledge, scientific literature reports no direct photochemical process which would uniquely involve the building blocks of the conjugated skeleton leading to the disruption of $\pi$-conjugation. The side-chain cleavage hypotheses presented in the literature for the degradation of P3HT and PCDTBT cannot, however, be validated for PDTSTzTz uniquely on the basis of UV-vis and IR analyses presented herein.

In order to clarify the mechanisms occurring in the early stages of polymer degradation and investigate the possible sidechain cleavage processes, intermediate short-lived free radicals formed during the irradiation process have been studied herein by performing spin trapping experiments.

3.1.2 EPR experimental data. In the present study, the spin trap used was the DMPO nitrone, which results in a stable nitroxide spin adduct; the EPR hyperfine couplings of which allow the nature of the trapped radicals to be identified. The spin trapping method using DMPO was applied to study the free radicals formed after $15 \mathrm{~min}$ of irradiation of the polymer wetted by a solution of DMPO in ethanol. The spectra of ethanol with DMPO, with and without irradiation under inert atmosphere, have been recorded; no signals were observed. Similarly, the spectra of the polymer without DMPO, with and without irradiation under Ar, were recorded: again no EPR signals were observed in either case (Fig. 4a presents the EPR spectrum of the polymer, without DMPO). Subsequently, the polymer without DMPO, in the presence of oxygen, with and without illumination was characterized. In dark conditions, no signal was observed, while under illumination, a stable signal exhibiting a single line (Fig. 4b) with a $g$-factor equal to 2.0023 was recorded.

Accordingly, the spectrum of PDTSTzTz wetted by the DMPO solution and illuminated under argon was then recorded. Fig. 4c presents the recorded spectrum together with its simulation created with a single radical species using three hyperfine couplings: $\mathrm{An}=15.2 \mathrm{G} ; \mathrm{AH}_{1}=22 \mathrm{G}$ and $\mathrm{AH}_{2}=0.5 \mathrm{G}$. These hyperfine couplings are very similar to those observed for an
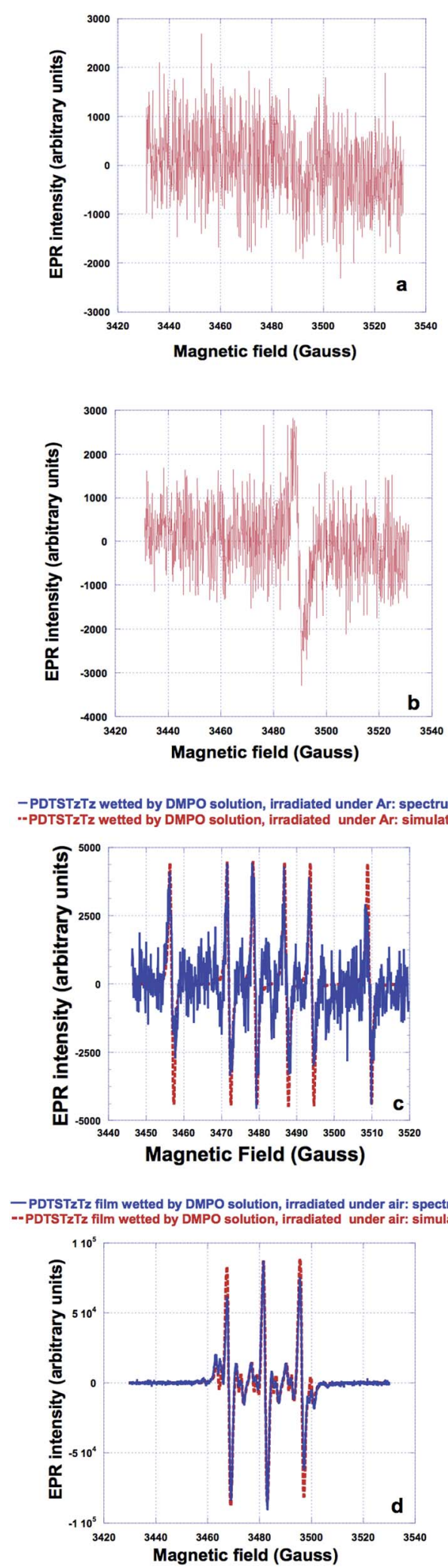

Fig. 4 EPR spectra of PDTSTzTz not wetted by DMPO solution, and irradiated with the solar simulator (a) under argon and; (b) in the presence of air $(g=2.0023)$. EPR spectra of PDTSTzTz wetted by DMPO solution and irradiated (c) under argon; (d) in presence of air. Parts (c) and (d) show the experimental spectra (solid blue line) together with their simulations (dotted red line). 
alkyl radical of $\mathrm{R}^{\cdot}$ type (for example $\mathrm{CH}_{3} \mathrm{CH}_{2}{ }^{\circ}$ in water gives rise to hyperfine couplings of $\left.\mathrm{AN}=16.3 \mathrm{G}, \mathrm{AH}_{1}=23.5 \mathrm{G}\right){ }^{28}+$ The fact that hyperfine couplings observed in our case are slightly lower is attributed to the difference in polarity between water and ethanol. Finally, the spectrum of PDTSTzTz, wetted by the DMPO solution, and irradiated under oxygen was recorded; Fig. 4d shows the experimental spectrum together with its simulation/fit. The experimental spectrum is satisfactorily fitted with two radicals: the first radical (of low intensity) gives rise to hyperfine couplings of $\mathrm{AN}=13.4 \mathrm{G}, \mathrm{AH}_{1}=7.5 \mathrm{G}$ and $\mathrm{AH}_{2}=$ $1.8 \mathrm{G}$ (six pairs of lines), while the second radical (of higher intensity) gives rise to only one hyperfine coupling of AN $=14 \mathrm{G}$. The hyperfine couplings of the first radical species are very similar to those observed for an alkoxy radical of the type $\mathrm{R}^{\prime} \mathrm{O}^{\cdot}$ (for example $\mathrm{CH}_{3} \mathrm{CH}_{2} \mathrm{O}^{\circ}$ in ethanol exhibits $\mathrm{AN}=13.5 \mathrm{G}, \mathrm{AH}_{1}=$ $7.4 \mathrm{G}$ and $\left.\mathrm{AH}_{2}=1.7 \mathrm{G}\right){ }^{28}$ The hyperfine AN coupling of the second radical fits well with that of the oxidative degradation product of DMPO.$^{28}$ Both species present a $g$-factor in the order of 2.006. The EPR data herein are interpreted as follows: first, the stable spectrum $(g=2.0023)$ observed for the polymer alone (without DMPO) when illuminated under oxygen is ascribed to the formation of the cation radical of the polymer associated with the superoxide anion radical, as previously reported for other polymers. ${ }^{29}$ Second, when irradiation of the polymer with DMPO is performed, the observed $\mathrm{R}^{\circ}$ signal (Fig. $4 \mathrm{~b}$ ) is attributed to a 'small' alkyl molecule and not to a macroradical. In fact, if this signal would correspond to a polymeric structure, the lines would be broader due to the rotational diffusion correlation time, especially the one located at high external magnetic field. ${ }^{30,31}$ Thirdly, when irradiation of the polymer with DMPO is performed under oxygen, the observed $\mathrm{R}^{\prime} \mathrm{O}^{\bullet}$ (Fig. 4b) results from the oxidation of alkyl radicals.

3.1.3 DFT calculations. Firstly, the $g$-factor of the polaron of PDTSTzTz was calculated. The determined value (2.0023) was in full agreement with that found by EPR (2.0023). As mentioned above, light absorption is able to provoke direct homolytic scission of weak bonds. Thus, we investigated two situations; one in which the DTS moiety loses a 2-ethyl-hexyl chain via direct homolysis of the $\mathrm{Si}-\mathrm{C}$ bond and the other in which the thiophene ring loses its lateral alkyl chain (a propyl group in this model, see Fig. 1). The bond dissociation energies were found to be 77.76 and $102.31 \mathrm{kcal} \mathrm{mol}^{-1}$, respectively. The magnitude of these values indicates that the $\mathrm{Si}-\mathrm{R}$ can be homolitically broken following the mechanism published for polyvinylcarbazole material. ${ }^{32,33}$ This is an interesting result, since the delocalization of the so-formed radical can take place partially within the heterocycle in question (as presented in Fig. 5), which considerably lowers the bond energy. It is noteworthy that the energy of UV photons is actually sufficient to break this weak bond (71-86 kcal mol${ }^{-1}$ for photons in the range of $330-400 \mathrm{~nm}$ ).

In addition to the homolytic scissions, irradiation of the sample in the presence of oxygen would also lead to photooxidative processes, commonly starting by abstraction of labile

\$ And references therein.

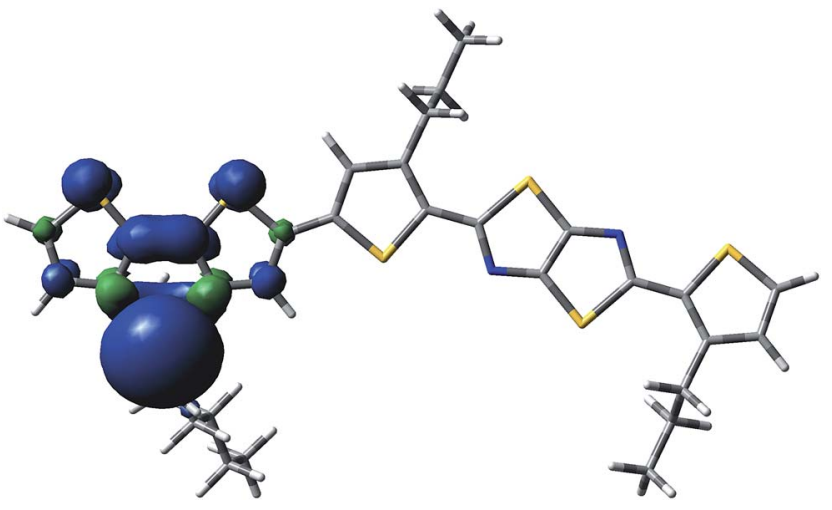

Fig. 5 Spin delocalization over the PDTSTzTz repeat unit which has lost the lateral chain of the Si atom moiety.

hydrogen atoms of the polymer as reported for PCDTBT ${ }^{9}$ and PVK. ${ }^{32,33}$ Using DFT calculations, the most labile hydrogen atoms of PDTSTzTz have been identified. The bond dissociation energies (BDEs) were calculated for the labelled protons in Fig. 1 and the values are given in Table 1.

From the data shown in Table 1, one can see that the hydrogen atom in the alpha position to the thiophene ring is the most labile one, and can therefore be more easily abstracted from the chain, even more so than the hydrogen on the tertiary carbon of the 2-ethyl-hexyl chain, in line with Santos Silva et al. ${ }^{23}$ Furthermore, the spin delocalization over the polymer repeat unit which has lost a $\mathrm{H}$ radical in the alphaposition of the thiophene units takes place over a large amount of neighbouring double bonds as presented in Fig. 6 . Therefore, although the energy of dissociation of the $\mathrm{C}-\mathrm{H}$ bond is larger than that of $\mathrm{Si}-\mathrm{C}$ bond, this radical is more delocalized, thus, more stable.

\subsection{Discussion}

The UV-vis absorbance decay of PDTSTzTz films indicate a higher degree of degradation for samples irradiated in air. Thus, whilst pure photolytic processes can happen both in the presence and absence of oxygen, photooxidative processes are those predominantly leading to the degradation of the polymer.

Table 1 UBL3YP/6-31G** energetic $\mathrm{C}-\mathrm{H}$ bonds calculations (kcal $\mathrm{mol}^{-1}$ ) in PDTSTzTz

\begin{tabular}{lc}
\hline Label & BDE $\left(\mathrm{kcal} \mathrm{mol}^{-1}\right)$ \\
\hline $\mathrm{a}$ & 100.18 \\
$\mathrm{~b}$ & 97.43 \\
$\mathrm{c}$ & 101.45 \\
$\mathrm{~d}$ & 103.92 \\
$\mathrm{e}$ & 104.63 \\
$\mathrm{f}$ & 108.76 \\
$\mathrm{~g}$ & 101.26 \\
$\mathrm{~h}$ & 106.98 \\
$\mathrm{i}$ & 89.93 \\
$\mathrm{j}$ & 104.42
\end{tabular}




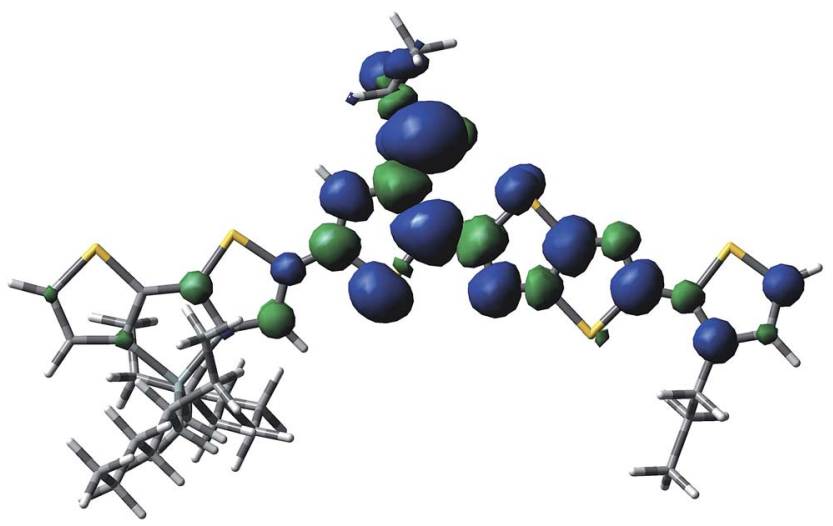

Fig. 6 Spin delocalization over the PDTSTzTz repeat unit which has lost a $\mathrm{H}$ radical in the alpha-position of the thiophene units.

As for photolytic processes (i.e. processes occurring upon irradiation under inert atmosphere), DFT calculations indicate that the Si-R bond can be homolitically broken. Such photochemical homolysis would lead to the formation of two types of radicals: a macro-Si" radical and a ${ }^{\circ} \mathrm{C}-\mathrm{R}$ radical, with the latter being unstable as delocalization is not possible (see Fig. 7). This result leads one to assume that the ${ }^{\circ} \mathrm{C}-\mathrm{R}$ trapped by DMPO under illumination without oxygen, observed by EPR, is likely to originate from the homolysis of the alkyl chain linked to the $\mathrm{Si}$ atom, the so-called $\mathrm{R}_{2}^{\cdot}$ according to the labelling in Fig. 1; with the calculated $g$-value for one DMPO adduct being in full agreement with that observed by EPR $(g=2.006)$. No spectrum corresponding to the macro- $\mathrm{Si}^{\circ}$ radical was observed from the present spin trapping experiments. This is not surprising since, to our knowledge, no adduct of DMPO with $\mathrm{Si}^{\circ}$ has ever been reported in the literature. The so-formed alkyl radicals could saturate the $\pi$-conjugated skeleton of the polymer (as suggested for $\mathrm{P} 3 \mathrm{HT})^{27}$ and the macroradicals formed on the Si atom are also susceptible to combination, thus provoking the crosslinking of the irradiated material (as suggested for PCDTBT $^{9}$ and PVK) ${ }^{32,33}$. Both processes could be responsible for the change in chemical structure of the polymer leading to a decrease in the UV-vis absorption of the polymer.

Under photooxidative conditions, radicals (such as those created by solely homolytic processes) are able to propagate the chain oxidation process by preferentially abstracting the most labile hydrogen atoms, i.e. those in the alpha position to the thiophene ring according to the data obtained by DFT calculations (Table 1). Irradiation of PDTSTzTz films in air led to EPR identification of the positive polaron (macro- $\mathrm{C}^{+\cdot}$ in Fig. 7 ) and of alkoxy radicals, in good accordance with these calculations. As depicted in Fig. 7, such alkoxy radicals can stem from the oxidation of the $\mathrm{R}_{1}$ radical $\left(\mathrm{R}_{1} \mathrm{O}^{\circ}\right)$ or the aforementioned $\mathrm{R}_{2}^{\cdot}$, generated by homolytical scission. Although the EPR spectrum in Fig. 4d has signals ascribed to both $\mathrm{R}_{1} \mathrm{O}^{\circ}$ and $\mathrm{R}_{2} \mathrm{O}^{*}$ (their adducts with DMPO giving similar EPR spectra), evolution and kinetics obtained by UV-vis and IR analysis clearly show that oxidative processes are predominant over intrinsic photochemical processes. Therefore, one can assume that $\mathrm{RO}^{\circ}$ are primarily related to $\mathrm{R}_{\mathbf{1}}$ moieties.

In this work, spin-trapping EPR spectroscopy is shown as a very sensitive tool, even after very short irradiation times (only 15 min AM 1.5 exposure), to identify the radical species (stable and unstable) resulting from photochemical processes. In combination with DFT calculations, the most likely photochemical processes occurring at these early stages of degradation are summarised in Fig. 7. According to the results herein presented, the absorption of UV-vis light by PDTSTzTz leads to (at least in part) the homolysis of the bond between the $\mathrm{Si}$ atom and its side-chain. Interestingly, previous investigations have indicated that replacing the $\mathrm{C}$ atom by $\mathrm{Si}$, as a bridging atom to the alkyl-chains, actually enhances the stability of the attached side-chain against photooxidation processes ${ }^{11}$ However, according to the study herein presented,

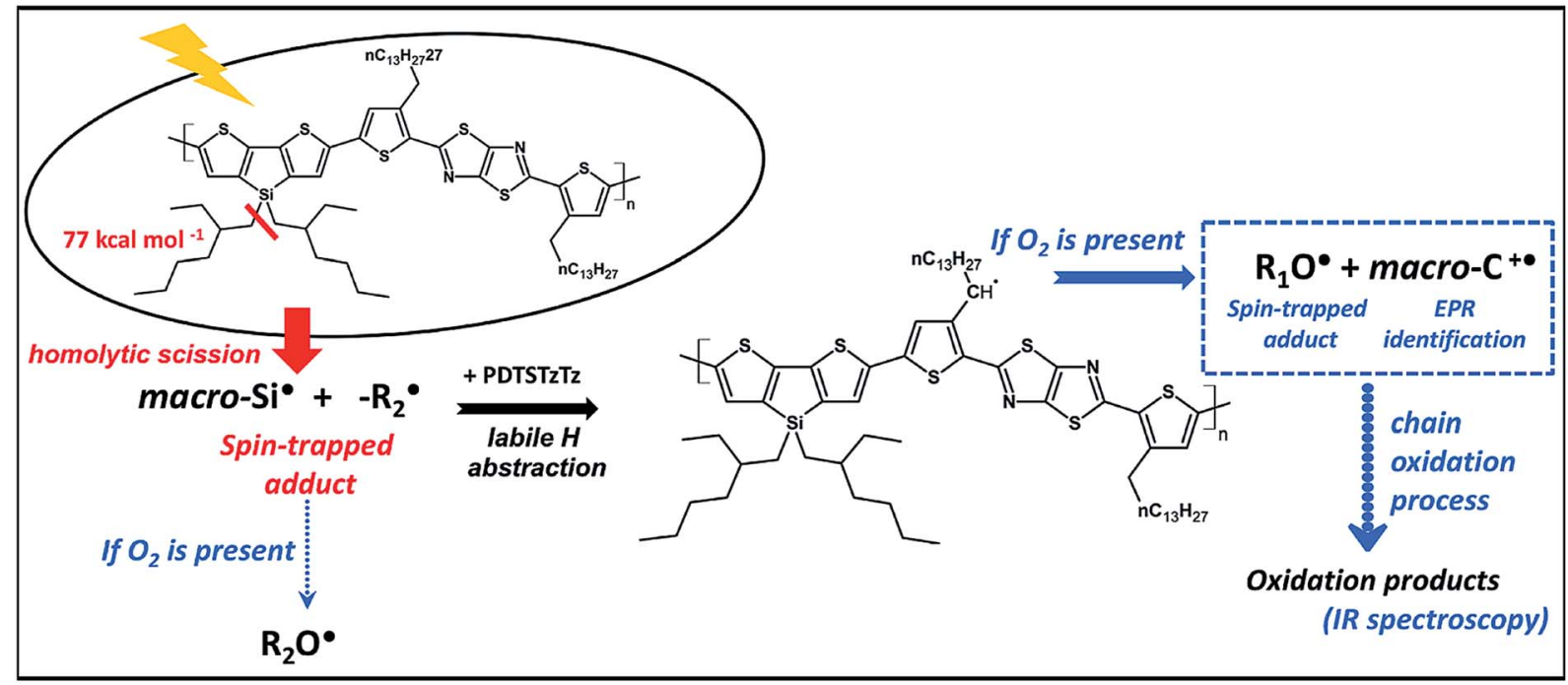

Fig. 7 Schematic representation of the early stages of the light-induced degradation of the PDTSTzTz polymer. 
the presence of a Si atom bearing a side-chain does represent the Achilles' heel of the chemical structure of PDTSTzTz; on the one hand, direct degradation can occur at this point under inert conditions via light-induced homolysis of this bond. On the other hand, if oxygen is present, the radicals produced are able to propagate oxidation, thus further degrading the polymer. More generally, irradiation in presence of oxygen is identified as the most destructive for the polymer. DFT calculations demonstrate that the primary steps of this oxidation process occur at the side chain attached to the thiophene ring, characterised by a low $\mathrm{C}-\mathrm{H}$ binding energy (see Fig. 7). In summary, the work presented identifies the combination between EPR and DFT calculations as a smart tool to identify the species at the origin of degradation. Furthermore, our findings are highly relevant for the organic electronic and photovoltaic communities as for designing polymers with more stable chemical structures.

\section{Conclusions}

Most polymers are photo-instable, and the identification of their Achilles' heel is particularly difficult when focusing on complex chemical structures such as LBG polymers. To this end we have demonstrated in the present study that the judicious combination of EPR spectroscopy and DFT calculations is a smart and efficient approach to unambiguously determine radical processes occurring in the early stages of photodegradation of low band gap polymers. Implementing this tool on PDTSTzTz, as a model polymer, we have revealed that it is unstable under light irradiation, as most polymers are, to identify the chemical structure of transient radicals formed during the photodegradation process and consequently to point out the Achilles' heel of the polymer structure. As follow up to this work, DFT modelling could allow one to tune the effect of structural modifications (for instance, carbon and germanium-analogue structures) to design a more photostable material. More generally, the smart combined approach demonstrated herein is a way to intuitively expand the tool box of building blocks available to design photostable low band gap polymers, which is a prerequisite for materials which will be submitted to UV-vis light irradiation over the lifetime of the organic solar cell.

\section{Acknowledgements}

The research leading to these results has received funding from the European Union Seventh Framework Programme (FP7/ 2011) under Grant Agreement ESTABLIS No. 290022.

\section{References}

1 M. Jørgensen, K. Norrman, S. A. Gevorgyan, T. Tromholt, B. Andreasen and F. C. Krebs, Adv. Mater., 2012, 24, 580-612. 2 S. Chatani, C. J. Kloxin and C. N. Bowman, Polym. Chem., 2014, 5, 2187-2201.

3 Y.-W. Su, S.-C. Lan and K.-H. Wei, Mater. Today, 2012, 15, 554-562.
4 P.-L. T. Boudreault, A. Najari and M. Leclerc, Chem. Mater., 2010, 23, 456-469.

5 H. Zhou, L. Yang and W. You, Macromolecules, 2012, 45, 607632.

6 H. Van Mullekom, J. Vekemans, E. Havinga and E. Meijer, Mater. Sci. Eng., R, 2001, 32, 1-40.

7 S. Karuthedath, T. Sauermann, H.-J. Egelhaaf, R. Wannemacher, C. J. Brabec and L. Lüer, J. Mater. Chem. A, 2015, 3, 3399-3408.

8 L. Müller-Meskamp, J. Fahlteich and F. Krebs, Stab. Degrad. Org. Polym. Sol. Cells, 2012, 269-329.

9 A. Tournebize, A. Rivaton, J.-L. Gardette, C. Lombard, B. Pépin-Donat, S. Beaupré and M. Leclerc, Adv. Energy Mater., 2014, 4, 1301530.

10 A. Tournebize, J.-L. Gardette, C. Taviot-Guého, D. Bégué, M. A. Arnaud, C. Dagron-Lartigau, H. Medlej, R. C. Hiorns, S. Beaupré, M. Leclerc, et al., Polym. Degrad. Stab., 2015, 112, 175-184.

11 I. Fraga Domínguez, P. D. Topham, P.-O. Bussière, D. Bégué and A. Rivaton, J. Phys. Chem. C, 2015, 119, 2166-2176.

12 E. G. Janzen and B. J. Blackburn, J. Am. Chem. Soc., 1968, 90, 5909-5910.

13 E. G. Janzen and B. J. Blackburn, J. Am. Chem. Soc., 1969, 91, 4481-4490.

14 C. Lagercrantz and S. Forshult, Trapping of free radicals formed by $\gamma$-irradiation of organic compounds, Univ. of goteborg technical report, 1968.

15 L. Chen, J. Mizukado, Y. Suzuki, S. Kutsuna, Y. Aoyama, Y. Yoshida and H. Suda, Chem. Phys. Lett., 2014, 605, 98-102. 16 D. R. Duling, J. Magn. Reson., Ser. B, 1994, 104, 105-110.

17 A. D. Becke, J. Chem. Phys., 1993, 98, 5648-5652.

18 C. Lee, W. Yang and R. G. Parr, Phys. Rev. B, 1988, 37, 785.

19 P. Stephens, F. Devlin, C. Chabalowski and M. J. Frisch, J. Phys. Chem., 1994, 98, 11623-11627.

20 K. Kim and K. Jordan, J. Phys. Chem., 1994, 98, 10089-10094.

21 W. J. Hehre, R. Ditchfield and J. A. Pople, J. Chem. Phys., 1972, 56, 2257-2261.

22 M. M. Francl, W. J. Pietro, W. J. Hehre, J. S. Binkley, M. S. Gordon, D. J. DeFrees and J. A. Pople, J. Chem. Phys., 1982, 77, 3654-3665.

23 H. S. Silva, A. Tournebize, D. Bégué, H. Peisert, T. Chassé, J.-L. Gardette, S. Therias, A. Rivaton and R. Hiorns, RSC Adv., 2014, 4, 54919-54923.

24 W. Kutzelnigg, U. Fleischer and M. Schindler, Deuterium and shift calculation, Springer, 1990, pp. 165-262.

25 F. Neese, Wiley Interdiscip. Rev.: Comput. Mol. Sci., 2012, 2, 73-78.

26 A. Rivaton, A. Tournebize, J. Gaume, P.-O. Bussière, J.-L. Gardette and S. Therias, Polym. Int., 2014, 63, 13351345.

27 M. Manceau, A. Rivaton, J.-L. Gardette, S. Guillerez and N. Lemaître, Sol. Energy Mater. Sol. Cells, 2011, 95, 13151325.

28 G. R. Buettner, Free Radicals Biol. Med., 1987, 3, 259-303.

29 Y. Aoyama, T. Yamanari, T. N. Murakami, T. Nagamori, K. Marumoto, H. Tachikawa, J. Mizukado, H. Suda and Y. Yoshida, Polym. J., 2015, 47, 26-30. 
30 A. Jeunet, B. Nickel and A. Rassat, Nouv. J. Chim., 1986, 10, 123-132.

31 Y. Xia, Y. Li, A. O. Burts, M. F. Ottaviani, D. A. Tirrell, J. A. Johnson, N. J. Turro and R. H. Grubbs, J. Am. Chem. Soc., 2011, 133, 19953-19959.
32 A. Tournebize, P.-O. Bussière, P. Wong-Wah-Chung, S. Thérias, A. Rivaton, J.-L. Gardette, S. Beaupré and M. Leclerc, Adv. Energy Mater., 2013, 3, 478-487.

33 A. Rivaton, B. Mailhot, G. Derderian, P. Bussiere and J.-L. Gardette, Macromolecules, 2003, 36, 5815-5824. 